\title{
Salvage endoscopic resection as a treatment for locoregional failure or recurrence following chemoradiotherapy or radiotherapy for esophageal cancer
}

\author{
RIEKO NAKAMURA, TAI OMORI, HIROYA TAKEUCHI, HIROFUMI KAWAKUBO, \\ TSUNEHIRO TAKAHASHI, NORIHITO WADA, YOSHIRO SAIKAWA and YUKO KITAGAWA \\ Department of Surgery, School of Medicine, Keio University, Tokyo 160-8582, Japan
}

Received March 13, 2015; Accepted April 1, 2016

DOI: $10.3892 / \mathrm{ol} .2016 .4478$

\begin{abstract}
Radiotherapy (RT) or chemoradiotherapy (CRT) is a potentially curative, non-surgical treatment option for esophageal cancer, although the rate of local failure within the esophagus remains relatively high. Salvage esophagectomy is not regarded as a common treatment for esophageal cancer, since it is a high-risk surgery with a relatively high surgical mortality rate. Salvage endoscopic resection (ER) for local failure is used for treatment when esophageal cancer is localized and superficial. To evaluate to usefulness of salvage ER, the present study reviewed the clinicopathological records and follow-up data of 37 patients that underwent salvage ER for esophageal cancer, following initial treatment with RT or CRT. Salvage ER was conducted on a total of 78 lesions observed in the 37 patients. Since a thick epithelium and lack of normal vessels on the surface of the mucosa are characteristics of esophageal mucosa following RT or CRT, almost all the lesions were detected using iodine dyeing, and not by narrow band imaging. The growth rate of the detected lesions was relatively high, and early treatment was required. No particular complications occurred during the endoscopic treatment. A total of 11 patients survived for $>5$ years subsequent to initial endoscopic treatment. Only 4 patients succumbed to esophageal cancer. In conclusion, the present study demonstrated that salvage ER following CRT or RT for esophageal cancer is a minimally invasive, safe, adaptive
\end{abstract}

Correspondence to: Dr Rieko Nakamura, Department of Surgery, School of Medicine, Keio University, 35 Shinanomachi, Shinjyuku-ku, Tokyo 160-8582, Japan

E-mail: rieko-na@cam.hi-ho.ne.jp

Abbreviations: RT, radiotherapy; CRT, chemoradiotherapy; ER, endoscopic resection; NBI, narrow band imaging; EMR, endoscopic mucosal resection; ESD, endoscopic submucosal dissection; APC, argon plasma coagulation; $\mathrm{CR}$, complete response; PR, partial response

Key words: esophageal cancer, chemoradiotherapy, local failure, salvage endoscopic treatment and curative method for superficial lesions without distant metastases in patients with esophageal cancer with local failure following CRT or RT.

\section{Introduction}

The risk of esophageal cancer is reported to be strongly associated with alcohol flushing and drinking, which is associated with inactive aldehyde dehydrogenase-2, smoking and dietary habits (1-3). These risk factors are well known and screening via endoscopy is extremely useful for the detection of esophageal cancer in high-risk groups. Esophageal cancer has a poor prognosis if advanced cancer is diagnosed, but the prognosis of patients with superficial esophageal cancer is acceptable. The 5-year survival rate has been reported to be $80.7 \%$ for stage I patients and $8.5 \%$ for stage IV patients (4).

As an initial treatment, curative endoscopic resection (ER) may be selected if the esophageal cancer is superficial, which is associated with extremely low risk complications, while radical surgery or definitive chemoradiotherapy (CRT) is used in advanced esophageal cancer cases to achieve a cure. Due to the high rate of surgical complications, CRT or radiotherapy (RT) is often used for esophageal cancer as a non-surgical treatment option $(5,6)$. Previous studies have demonstrated that definitive CRT for esophageal cancer is associated with a high response and survival rate (7-9), although local failure following CRT or RT remains a major challenge in achieving a cure. A standard treatment strategy for local failure following CRT or RT has not yet been determined, although a salvage esophagectomy is generally indicated for these cases. However, due to the technical challenge of radical surgery and the high rate of complications, salvage surgery has not yet been accepted as a standard treatment strategy for local failure. For local disease control, salvage ER may be utilized if the depth of invasion is limited to the mucosa or submucosa. Salvage ER is associated with a low risk of complications and may be used to achieve a cure if recurrences are local and superficial (10). The present authors have performed ER for such locoregional lesions and metachronous lesions appearing following CRT or RT. The present study reviewed the clinicopathological records and follow-up data for 37 patients that underwent salvage ER following CRT 
Table I. Histopathological characteristics of 37 patients with esophageal cancer.

\begin{tabular}{|c|c|}
\hline Characteristics & Value \\
\hline Total, n (\%) & $37(100.0)$ \\
\hline \multicolumn{2}{|l|}{ Gender, n (\%) } \\
\hline Male & $36(97.3)$ \\
\hline Female & $1(2.7)$ \\
\hline \multicolumn{2}{|l|}{ Age, years } \\
\hline Median & 71.5 \\
\hline Range & $53-85$ \\
\hline \multicolumn{2}{|c|}{ TNM stage, $\mathrm{n}(\%)$} \\
\hline I & $28(75.7)$ \\
\hline II & $2(5.4)$ \\
\hline III & $4(10.8)$ \\
\hline IV & $3(8.1)$ \\
\hline \multicolumn{2}{|c|}{ Depth of tumor invasion, $\mathrm{n}(\%)$} \\
\hline EP & $3(8.1)$ \\
\hline LPM & $6(16.2)$ \\
\hline MM & $5(13.5)$ \\
\hline SM1 & $2(5.4)$ \\
\hline $\mathrm{SM} 2$ & $3(8.1)$ \\
\hline SM3 & $5(13.5)$ \\
\hline SM & $5(13.5)$ \\
\hline MP & $1(2.7)$ \\
\hline $\mathrm{AD}$ & $3(8.1)$ \\
\hline AI & $4(10.8)$ \\
\hline \multicolumn{2}{|c|}{ Initial treatment method, $\mathrm{n}(\%)$} \\
\hline CRT & $27(73.0)$ \\
\hline RT & $10(27.0)$ \\
\hline \multicolumn{2}{|c|}{ Effect of initial treatment, n (\%) } \\
\hline CR & $22(59.5)$ \\
\hline PR & $15(40.5)$ \\
\hline
\end{tabular}

Age is at initial endoscopic treatment. TMN, tumor-node-metastasis; EP, epithelial; LPM, lamina propria; MM, muscularis mucosa; SM, submucosal; MP, muscularis propria; AD, adventitia; AI, adjacent organ invasion; CRT, chemoradiotherapy; RT, radiotherapy; CR, complete response; PR, partial response.

or RT for esophageal cancer to identify the outcome of these patients.

\section{Patients and methods}

Patients. Between January 2001 and December 2012 , 37 patients with esophageal cancer (total of 78 lesions) underwent salvage endoscopic treatment for recurrences or residual/metachronous lesions following definitive CRT or RT at the Department of Surgery, Keio University Hospital (Tokyo, Japan). The clinicopathological records of these patients, including pretreatment diagnoses, methods used for initial therapy, pre-salvage endoscopic treatment diagnoses, methods used for endoscopic treatment, complications, histological
Table II. Final pathological findings of all locoregional lesions that underwent ER, including depth of invasion.

A, Endoscopic therapy

\begin{tabular}{lc}
\hline Method & Lesions, $\mathrm{n}(\%)$ \\
\hline Total & $78(100.0)$ \\
EMR/ESD & $67(85.9)$ \\
APC & $11(14.1)$ \\
\hline
\end{tabular}

$\mathrm{B}$, Tumor invasion

Characteristic

Lesions, n (\%)

Total

$67(100.0)$

Diagnosis

Dysplasia $\quad 6(8.9)$

EP

$26(38.8)$

LPM

$17(25.3)$

$\mathrm{MM}$

$5(7.4)$

SM1

$6(8.9)$

SM2

$5(7.4)$

SM3

$1(1.4)$

SM

1 (1.4)

Lymphatic/vascular invasion

Positive margin

$15(22.3)$

Lateral (+)

$13(19.4)$

Vertical (+)

EMR, endoscopic mucosal resection; ESD, endoscopic submucosal dissection; APC, argon plasma coagulation; EP, epithelial; LPM, lamina propria; MM, muscularis mucosa; SM, submucosal.

features of resected specimens and outcomes, were examined by the present study.

\section{Results}

Clinicopathological diagnoses and initial treatment methods. The histopathological features of the patients are presented in Table I. The stage distribution of the patients was as follows, according to the tumor-node-metastasis (TNM) classification defined by the UICC (11): Stage I, 28 patients (75.6\%); stage II, 2 patients (5.4\%); stage III, 4 patients $(10.8 \%)$; and stage IV, 3 patients $(8.1 \%)$. The depth of tumor invasion of the patients was as follows, according to diagnosis by endoscopy and fluoroscopy: Epithelial (EP)/lamina propria (LPM), 9 patients (24.3\%); muscularis mucosa (MM)/submucosal (SM) 1, 7 patients (18.9\%); SM2/SM, 8 patients $(21.6 \%)$; and muscularis propria (MP)/deeper than MP, 8 patients $(21.6 \%)$. Table II reveals the initial methods of treatment of the patients; 27 patients $(72.9 \%)$ underwent CRT and 10 patients $(27.0 \%)$ underwent RT. Overall, 22 patients (59.4\%) exhibited a complete response (CR) and 15 patients $(40.5 \%)$ exhibited a partial response. 

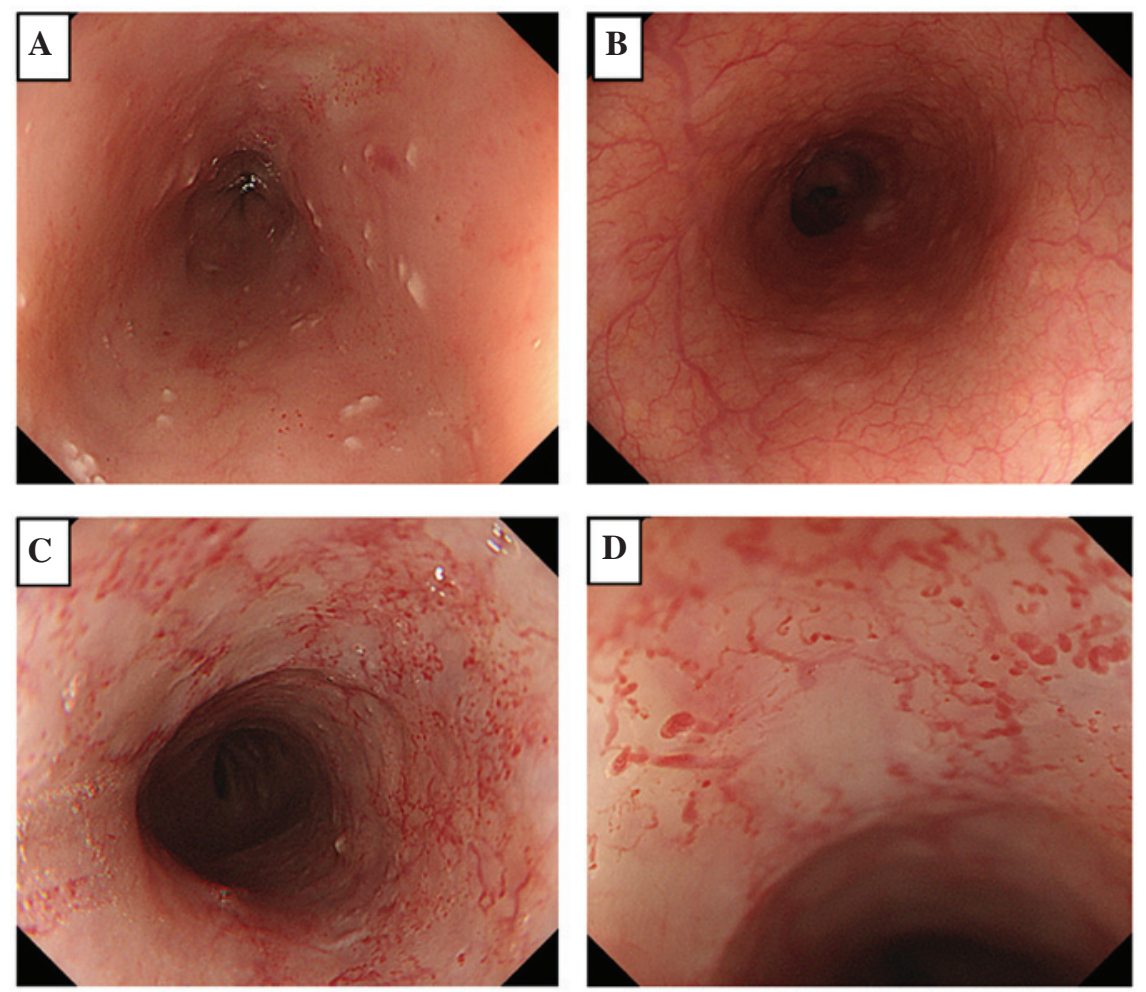

Figure 1. (A) Appearance of esophageal mucosa without a disease lesion following CRT. (B) Normal appearance of esophageal mucosa. (C) Severe radiation esophagitis following CRT and (D) magnified endoscopic appearance. CRT, chemoradiotherapy.

Characteristics of locoregional failure or recurrence. The esophageal mucosa following CRT or RT is often characterized by a thick epithelium and the disappearance of normal superficial vessels in the mucosa (Fig. 1A) compared with normal esophageal mucosa (Fig. 1B). Radiation esophagitis causes the appearance of abnormal vessels in the esophageal mucosa (Fig. 1C and D) and renders the detection of cancer lesions, using white light imaging or narrow band imaging (NBI), relatively challenging. In the present study, almost all the lesions were detected using iodine staining, and the most important characteristic was the speed of tumor growth following the first detection of cancer lesions, which was evaluated by endoscopy. If lesions that could not be confirmed as cancer were detected, a re-examination was performed within a relatively short period (2-3 months later) (Fig. 2A and B).

Methods of treatment for locoregional failure or recurrence following CRT or RT. A total of 78 lesions were detected and treated in the 37 patients following CRT or RT. Endoscopic mucosal resection (EMR) or endoscopic submucosal dissection (ESD) was performed for 67 lesions (85.8\%), and argon plasma coagulation was performed for 11 lesions (14.1\%) (Table IIA). No serious complications occurred post-treatment.

Challenges associated with endoscopic therapy for locoregional failure or recurrence. Severe fibrous alterations of the submucosa may render the resection of the submucosa challenging and may increase the risk of perforation during ER in numerous cases (10). Furthermore, the total thickness due to radiation or ablation therapy may mean the cancer lesion is not detected definitively; therefore, residual cancer may occasionally recur and invade to a deeper layer (10). Consequently, the ESD technique requires a high level of endoscopic proficiency. Fig. $2 \mathrm{C}$ and D reveals treatment with ESD for a locoregional failure lesion following CRT for esophageal and hypopharyngeal duplication cancer. In this patient, the esophageal lesion exhibited a high-grade of CRT-induced fibrosis, making it challenging to detect the submucosal layer. To overcome this difficulty, the use of a 'clip with line' device developed by Oyama (11) simplified the dissection of the submucosa by providing a counter-traction for the cutting layer. This device not only improves the safety of the submucosa dissection, but also shortens the time required to perform the submucosal dissection during ESD.

Treatment courses for locoregional lesions following CRT and $R T$. Fig. 3 shows the treatment courses administered to patients for locoregional lesions following CRT/RT. Initial salvage ER was conducted for 40 lesions in 37 patients; 29 of these lesions were residual or recurrent lesions, and 11 were metachronous. Second-line or greater ER was conducted for 38 lesions occurring following initial ER in 18 patients; 22 of these lesions were residual or recurrent lesions and 16 were metachronous. In total, 19 patients did not develop recurrent, residual or metachronous lesions after initial salvage ER during the follow-up period.

Pathological diagnoses of ER-conducted specimens. Table IIB shows the pathological findings for all the locoregional lesions that were treated with ER, including the depth of invasion. A total of 49 lesions (62.8\%) were confirmed to exhibit dysplasia or EP/LPM, 11 lesions (14.1\%) were confirmed as MM/SM1, 

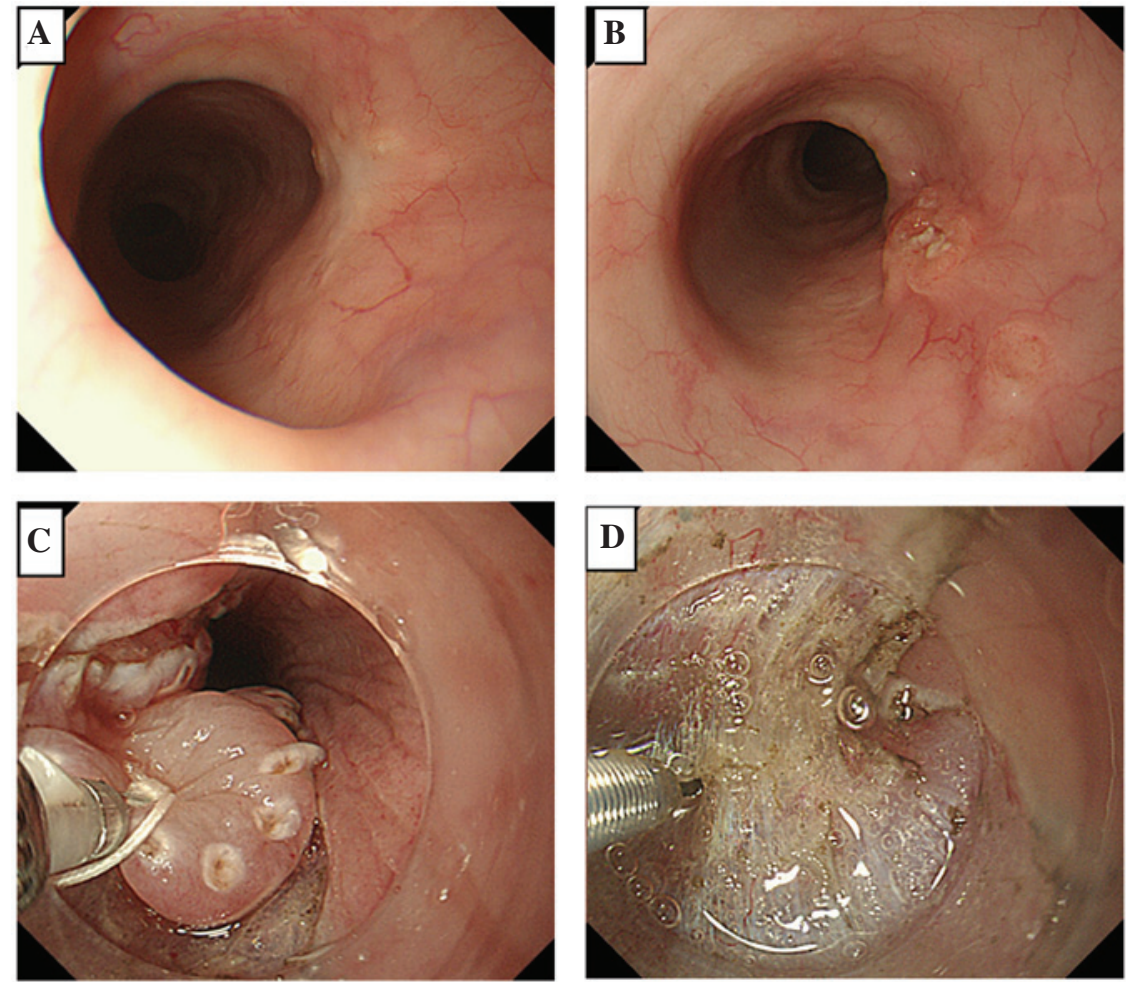

Figure 2. (A) Disease lesion following chemoradiotherapy (biopsies of lesions following iodine dyeing demonstrated no pathological signs of malignancy). and (B) endoscopic findings 3 months later, which demonstrated that the disease lesion was increased. (C and D) Salvage endoscopic submucosal dissection. (C) Equipping of 'clip with line' after entire circumference incision, and (D) incision of submucosal layer.



Figure 3. Treatment courses for locoregional lesions detected following CRT/RT. CRT, chemoradiotherapy; RT, radiotherapy.

and 7 lesions $(8.9 \%)$ were confirmed as SM2/SM3 or SM, according to the TNM staging classification (12). The majority of the lesions were T1a (mucosal lesion, early cancer of esophagus), and ER was a useful method for achieving a cure in patients with these lesions.

Patient outcomes following salvage ER. Table III shows the patient outcomes of 37 salvage ER cases. In total, 16 patients (43.2\%) were alive at the last follow-up and 11 patients survived for $\geq 5$ years. Only 4 patients succumbed to esophageal cancer, while 9 patients succumbed to other causes. Among the 4 patients that succumbed to esophageal cancer, the pretreatment diagnoses prior to CRT or RT were T1b for 3 patients and T3 for 1 patient, while the tumor depths at ER were $2 \mathrm{EP}, 1 \mathrm{SM} 1$ and $1 \mathrm{SM} 2$. A total of 8 patients were lost to follow-up.

\section{Discussion}

Esophageal cancer has a good prognosis if the disease is detected as a superficial lesion that may be treated with ER and has an EP or LPM depth of invasion. However, chemotherapy is recommended for local advanced lesions or patients with regional lymph node or distant metastases, since this type of disease is known to be aggressive (13). Patients with unresectable advanced cancer that have never been referred for surgical resection, have refused surgery or have characteristics that make them ineligible for surgery, including poor performance status, are candidates for definitive CRT or RT. However, the frequency of a pathological CR to CRT ranges between 30 and $62 \%(7-9,14,15)$, and the majority of recurrences reportedly occur within the radiation field when examined either retrospectively or prospectively (16-18). Additionally, 
esophageal cancer often occurs as metachronous or multiple cancers (19). At the Keio University Hospital, regular endoscopic examinations are performed to detect not only recurrent lesions following RT or CRT, but also novel superficial cancer lesions in radiation fields. As discussed, in the majority of CRT or RT cases, normal regular vessels are not visible through the mucosa and cancer lesions cannot be detected as brown areas using NBI. However, iodine dyeing is extremely useful for detecting lesions; therefore, it should be included in regular examinations of patients that have undergone CRT or RT. In cases where superficial lesions are detected and no distant metastases are apparent, ER treatment appears to be a useful method for obtaining local control.

Salvage esophagectomy is one option for the treatment of local recurrence or locoregional lesions, although a salvage esophagectomy following CRT reportedly has a high risk of complications, a high mortality rate and there is an increased challenge of treatment for recurrences following salvage surgery $(20,21)$. Regarding complications, the incidence of conduit necrosis has been reported to be as high as $25 \%$ following salvage esophagectomy $(22,23)$, and the creation of an anastomosis within the radiated field in the chest has been demonstrated to result in a higher than acceptable leakage rate (24). Regarding patient outcome, previous studies have reported long-term survival rates of $50 \%$ at 3 years and $30-35 \%$ at 5 years $(25,26)$ following salvage esophagectomy.

By contrast, the major complications of salvage endoscopic treatments are bleeding and perforation, and the majority of those complications are treated by endoscopy or careful observation with conservative follow-up care. No severe complications were experienced in the present patient series, and none of the patients required surgery due to a complication or succumbed to a complication. However, the mucosa exhibits severe scarring following RT or CRT, making it challenging to identify the submucosal layer and to cut the appropriate layer when performing an ER. Thus, this strategy requires a high level of technical proficiency and should only be performed by experienced surgeons, and careful follow-up is required.

According to the present results, among the 37 patients that underwent endoscopic treatment, the pretreatment diagnoses indicated that $75 \%$ of the patients were classified as stage I and $78 \%$ were estimated to have a T1 depth of SM tumor invasion. Pathologically, $80 \%$ of the resected specimens demonstrated EP to SM1 invasion. Salvage ER was well suited to the pretreatment diagnoses of T1 esophageal cancer, while $22 \%$ of the T2-T4 patients were able to undergo endoscopic treatment following tumor reduction by CRT or RT in cases where the tumor invasion of the final resected specimen proved to be $\mathrm{T} 1$ invasion.

At Keio University Hospital, CRT is conducted for patients that cannot undergo or have refused surgery. The aim is to avoid surgery as much as possible if recurrent, residual or metachronous lesions are detected following CRT. Salvage endoscopic treatment is conducted only in cases where local control is thought to be probable; thus, cases with distant metastases or lesions that have invaded deeper than the submucosal layer are not candidates for salvage ER. Regardless of the pathological results, typical follow-up care includes endoscopic and computed tomography examinations to confirm the absence of local lesions and distant metastases.
Table III. Outcomes of 37 patients that underwent salvage endoscopic resection.

\begin{tabular}{lr}
\hline Outcome & $\mathrm{n}(\%)$ \\
\hline Total & $37(100.0)$ \\
Alive & $16(43.2)$ \\
$\geq 5$ years & $11(29.7)$ \\
$<5$ years & $5(13.5)$ \\
Succumbed & $13(35.1)$ \\
Esophageal cancer & $4(10.8)$ \\
Other disease & $9(24.3)$ \\
Lost to follow-up & $8(21.6)$ \\
\hline
\end{tabular}

In addition, $48 \%$ of patients required second-line or additional endoscopic treatment following salvage ER of the first lesion; therefore, the regular examination for the early detection of cancer lesions and the early ER of these types of lesions is essential. Regardless of whether recurrent or metachronous cancer is present, early detection is the most important factor for good patient outcome.

Certain cases in the current patient series had laryngopharyngeal cancer in addition to esophageal cancer; 3 of these patients were diagnosed prior to CRT or RT for esophageal cancer, 4 patients were diagnosed at the same time as CRT or RT and 6 patients were diagnosed following CRT or RT. In addition, 6 patients were diagnosed with gastric cancer. Such high-risk cases often do not undergo surgery for esophageal cancer, and the esophageal cancer lesions do not necessarily affect the outcome of these patients. Similarly, surgery may not be performed in elderly patients, and the median age of the patients in this study was $>70$ years old. Therefore, salvage ER is well suited to cases with two or more cancers and elderly patients in whom surgery may not be well tolerated. In the present study, only 4 patients $(10.8 \%)$ succumbed to esophageal cancer and 11 patients $(29.7 \%)$ survived for $\geq 5$ years following their first endoscopic treatment. These results are thought to be acceptable for salvage treatment following the presence of recurrent or metachronous lesions.

Certain authors have reported excellent long-term survival without severe complications following salvage endoscopic treatment subsequent to CRT; 5-year survival rates following salvage endoscopic treatment have been reported as $36-49 \%(10,27)$. In the present study, no severe complications occurred following salvage endoscopic treatment, and only 4 patients succumbed to esophageal cancer, while 11 patients (29.7\%) survived for $\geq 5$ years. While salvage ER requires a high quality of diagnostic capability and advanced techniques, it should be considered as a treatment option for esophageal cancer to achieve a cure without surgery.

The present results demonstrated that salvage ER is an excellent strategy for obtaining long-term survival following local recurrence or metachronous lesions subsequent to CRT or RT for esophageal cancer. This technique may be used to excise the lesions completely, has a low risk of complications and is less stressful for patients, compared with salvage surgery. This strategy is well suited for patients whose treatment 
strategy may be uncertain, including patients with two or more cancers, a history of surgery or elderly patients. The present results were acceptable in terms of curability, patients' quality of life and patient outcome.

\section{References}

1. Cui R, Kamatani Y, Takahashi A, Usami M, Hosono N, Kawaguchi T, Tsunoda T, Kamatani N, Kubo M, Nakamura Y and Matsuda K: Functional variants in ADH1B and ALDH2 coupled with alchol and smoking synergistically enhance esophageal cancer risk. Gastroenterology 137: 1768-1775, 2009.

2. Yokoyama T, Yokoyama A, Kato H, Tsujinaka T, Muto M, Omori T, Haneda T, Kumagai Y, Igaki H, Yokoyama M, et al: Alcohol flushing, alcohol and aldehyde dehydrogenase genotypes, and risk for esophageal squamous cell carcinoma in Japanese men. Cancer Epidemiol Biomarkers Prev 12: 1227-1233, 2003.

3. Mitsumori T, Matsusaka T, Wakasugi K, Takenaka M, Kume K, Fujinaga $\mathrm{Y}$, Teraoka $\mathrm{H}$ and Iwashita A: A clinicopathological study of gastric cancer with special reference to age of the patients: An analysis of 1,630 cases. World J Surg 13: 225-230; discussion 230-231, 1989.

4. Japanese Society of Esophageal Diseases: Comprehensive registry of esophageal cancer in Japan, 1999. Esophagus 2: 43-69, 2005.

5. Ohtsu A: Chemoradiotherapy for esophageal cancer: Current status and perspectives. Int J Clin Oncol 9: 444-450, 2004.

6. Ajani J, Bekaii-Saab T, D'Amico TA, Fuchs C, Gibson MK, Goldberg M, Hayman JA, Ilson DH, Javle M, Kelley S, et al: Esophageal Cancer Clinical Practice Guidelines. J Natl Compr Canc Netw 4: 328-347, 2006

7. Ishihara R, Yamamoto $\mathrm{S}$, lishi H, Takeuchi Y, Sugimoto N, Higashino K, Uedo N, Tatsuta M, Yano M, Imai A and Nishiyama K: Factors predictive of tumor recurrence and survival after initial complete response of esophageal squamous cell carcinoma to definitive chemoradiotherapy. Int J Radiat Oncol Biol Phys 76: 123-129, 2010.

8. Kato K, Muto K, Minashi K, Ohtsu A, Ishikura S, Boku N, Takiuchi H, Komatsu Y, Miyata Y and Fukuda H; Gastrointestinal Oncology Study Group of the Japan Clinical Oncology Group (JCOG): Phase II study of chemoradiotherapy with 5-fluorouracil and cisplatin for stage II-III esophageal squamous cel carcinoma: JCOG trial (JCOG 9906). Int Radiat Oncol Biol Phys 81: 684-690, 2011.

9. Suzuki A, Xiao L, Hayashi Y, Blum MA, Welsh JW, Lin SH, Lee JH, Bhutani MS, Weston B, Maru DM, et al: Nomograms for prognostication of outcome in patients with esophageal and gastroesophageal carcinoma undergoing definitive chemoradiotherapy. Oncology 82: 108-113, 2012.

10. Yano T, Muto M, Hattori S, Minashi K, Onozawa M, Nihei K, Ishikura S, Ohtsu A and Yoshida S: Long-term results of salvage endoscopic mucosal resection in patients with local failure after definitive chemoradiotherapy for esophageal squamous cell carcinoma. Endoscopy 40: 717-721, 2008

11. Oyama T: Counter traction makes endoscopic submucosal dissection easier. Clin Endosc 45: 375-378, 2012

12. Edge SB, Byrd DR, Compton CC, Fritz AG and Greene FL (eds) AJCC Cancer Staging Manual. 7th edition. Springer-Verlag, New York, NY, 2010.

13. Kato $\mathrm{H}$ and Nakajima M: Treatments for esophageal cancer: $\mathrm{A}$ review. Gen Thorac Cardiovasc Surg 61: 330-335, 2013.
14. Michel P, Adenis A, Di Fiore F, Boucher E, Galais MP, Dahan L, Mirabel X, Hamidou H, Raoul JL, Jacob JH, et al: Induction cisplatin-irinotecan followed by concurrent cisplatin-irrinotecan and radiotherapy without surgery in oesophageal cancer: Multicenter phase II FFCD trial. Br J Cancer 95: 705-709, 2006

15. Conroy T, Yataghène Y, Etienne PL, Michel P, Senellart H, Raoul JL, Mineur L, Rives M, Mirabel X, Lamezec B, et al: Phase II randomised trial of chemoradiotherapy with FOLFOX4 or cisplatin plus fluorouracil in oesophageal cancer. $\mathrm{Br}$ J Cancer 103: 1349-1355, 2010.

16. Minsky BD, Pajak TF, Ginsberg RJ, Pisansky TM, Martenson J, Komaki R, Okawara G, Rosenthal SA and Kelsen DP: INT 0123 (Radiation Therapy Oncology Group 94-05) Phase III trial of combined-modality therapy for esophageal cancer: High-dose versus standard-dose radiation therapy. J Clin Oncol 20: 1167-1174, 2002.

17. Crosby TD, Brewster AE, Borley A, Perschky L, Kehagioglou P, Court $\mathbf{J}$ and Maughan TS: Definitive chemoradiation in patients with inoperable oesophageal carcinoma. Br J Cancer 90: 70-75, 2004.

18. Welsh J, Settle SH, Amini A, Xiao L, Suzuki A, Hayashi Y, Hofstetter W, Komaki R, Liao Z and Ajani JA: Failure patterns in patients with esophageal cancer treated with definite chemoradiation. Cancer 118: 2632-2640, 2012.

19. Kudou M, Shiozaki A, Fujiwara H, Kosuga T, Konishi H, Morimura R, Murayama Y, Komatsu S, Kuriu Y, Ikoma H, et al: Clinical analysis of esophageal cancer patients with a history of metachronous primary cancer. Gan To Kagaku Ryoho 41: 2030-2032, 2014

20. Swisher SG, Wynn P, Putnam JB, Mosheim MB, Correa AM, Komaki RR, Ajani JA, Smythe WR, Vaporciyan AA, Roth JA and Walsh GL: Salvage esophagectomy for recurrent tumors after definitive chemotherapy and radiotherapy. J Thorac Cardiovasc Surg 123: 175-183, 2002.

21. Meunier B, Raoul J, Le Prisé E, Lakéhal M and Launois B: Salvage esophagectomy agter unsuccessful curative chemoradiotherapy for squamous cell cancer of the esophagus. Dig Surg 15: 224-226, 1998.

22. Nakamura T, Hayashi K, Ota M, Eguchi R, Ide H, Takasaki K and Mitsuhashi N: Salvage esophagectomy after definitive chemotherapy and radiotherapy for advanced esophageal cancer. Am J Surg 188: 261-266, 2004

23. Amimi A, Ajani J, Komaki R, Allen PK, Minsky BD, Blum M, Xiao L, Suzuki A, Hofstetter W, Swisher S, et al: Factors associated with local-regional failure after definitive chemoradiation for locally advanced esophageal cancer. Ann Surg Oncol 21: 306-314, 2014

24. Juloori A, Tucker SL, Komaki R, Liao Z, Correa AM, Swisher SG, Hofstetter WL and Lin SH: Influence of preoperative radiation field on postoperative leak rates in esophageal cancer patients after trimodality therapy. J Thorac Oncol 9: 534-540, 2014.

25. Gardner-Thorpe J, Hadwick RH and Dwerryhous SJ: Salvage esophagectomy after local failure of definitive chemoradiotherapy. Br J Surg 94: 1059-1066, 2007.

26. D'Journo XB, Michelet P, Dahan L, Doddoli C, Seitz JF, Giudicelli R, Fuentes PA and Thomas PA: Indications and outcome of salvage surgery for esophageal cancer. Eur J Cardiothrorac Surg 33: 1117-1123, 2008.

27. Yano T, Muto M, Minashi K, Onozawa M, Nihei K, Ishikura S, Kaneko $\mathrm{K}$ and Ohtsu A: Long-term results of salvage photodynamic therapy for patients with local failure after chemoradiotherapy for esophageal squamous cell carcinoma. Endoscopy 43: 657-663, 2011. 